\title{
Asymptomatic Transmission of SARS-CoV-2 on Evacuation Flight
}

\author{
Sung Hwan Bae, Heidi Shin, Ho-Young Koo, Seung Won Lee, Jee Myung Yang, Dong Keon Yon
}

We conducted a cohort study in a controlled environment to measure asymptomatic transmission of severe acute respiratory syndrome coronavirus 2 on a flight from Italy to South Korea. Our results suggest that stringent global regulations are necessary for the prevention of transmission of this virus on aircraft.

U ndocumented cases of severe acute respiratory syndrome coronavirus 2 (SARS-CoV-2) infection have been common during the coronavirus disease (COVID-19) global pandemic (1-3). Although inflight transmission of symptomatic COVID-19 has been well established $(1,2)$, the evidence for transmission of asymptomatic COVID-19 on an aircraft is inconclusive. We conducted a cohort study evaluating asymptomatic passengers on a flight that carried 6 asymptomatic patients with confirmed SARSCoV-2 infections. The Institutional Review Board of Armed Force Medical Command approved the study protocol. The ethics commission waived written informed consent because of the urgent need to collect data on COVID-19.

\section{The Study}

On March 31, 2020, we enrolled in our study 310 passengers who boarded an evacuation flight from Milan, Italy, to South Korea. This evacuation flight was conducted under strict infection control procedures by the Korea Centers for Disease Control and Prevention (KCDC), based on the guidelines of the World

Author affiliations: Soonchunhyang University College of Medicine, Seoul, South Korea (S.H. Bae); Soonchunhyang University Seoul Hospital, Seoul (S.H. Bae); Harvard Business School, Boston, Massachusetts, USA (H. Shin); Korea University College of Medicine, Seoul (H.-Y. Koo); Sejong University College of Software Convergence, Seoul (S.W. Lee); University of Ulsan College of Medicine, Seoul (J.M. Yang); Asan Medical Center, Seoul (J.M. Yang); Armed Force Medical Command, Republic of Korea Armed Forces, Seongnam, South Korea (D.K. Yon); CHA University School of Medicine, Seongnam (D.K. Yon)

DOI: https://doi.org/10.3201/eid2611.203353
Health Organization (WHO) (4). When the passengers arrived at the Milan airport, medical staff performed physical examinations, medical interviews, and body temperature checks outside the airport before boarding, and 11 symptomatic passengers were removed from the flight. Medical staff dispatched from KCDC were trained in infection control under the guidance of the KCDC and complied with the COVID-19 infection protocol, which was based on WHO guidelines (4). N95 respirators were provided, and passengers were kept $2 \mathrm{~m}$ apart for physical distancing during preboarding. Most passengers wore the N95 respirators except at mealtimes and when using the toilet during the flight. After an 11-hour flight, 299 asymptomatic passengers arrived in South Korea and were immediately quarantined for 2 weeks at a government quarantine facility in which the passengers were completely isolated from one another. Medical staff examined them twice daily for elevated body temperature and symptoms of COVID-19. All passengers were tested for SARS-CoV-2 by reverse transcription PCR twice, on quarantine day 1 (April 2) and quarantine day 14 (April 15).

Asymptomatic patients were those who were asymptomatic when they tested positive and did not develop symptoms within 14 days after testing (5). Among the 299 passengers (median age 30.0 years; $44.1 \%$ male), 6 had a confirmed positive result for SARS-CoV-2 on quarantine day 1 and were transferred immediately to the hospital (Table). At 14 days after the positive test, the 6 patients reported no symptoms and were categorized as asymptomatic.

On quarantine day 14, a 28-year-old woman who had no underlying disease had a confirmed positive test result for COVID-19. On the flight from Milan, Italy, to South Korea, she wore an N95 mask, except when she used a toilet. The toilet was shared by passengers sitting nearby, including an asymptomatic patient. She was seated 3 rows away from the asymptomatic patient (Figure). Given that she did not go outside and had self-quarantined for 3 weeks alone at her home in Italy before the flight and did not use 
Table. Baseline characteristics and quarantine day 1 SARS-CoV-2 test results for asymptomatic passengers from flight from Milan, Italy, to South Korea, March 2020*

\begin{tabular}{|c|c|c|c|}
\hline Characteristics & $\begin{array}{c}\text { All asymptomatic } \\
\text { passengers, } N=299\end{array}$ & $\begin{array}{l}\text { Passengers testing negative } \\
\text { for SARS-CoV- } 2, n=293\end{array}$ & $\begin{array}{l}\text { Patients testing positive } \\
\text { for SARS-CoV-2, } n=6\end{array}$ \\
\hline Median age (IQR), y & $30.0(27.0-35.0)$ & $30.0(27.0-35.0)$ & $28.0(9.9-45.0)$ \\
\hline \multicolumn{4}{|l|}{ Sex } \\
\hline M & $132(44.1)$ & $128(43.7)$ & $4(66.7)$ \\
\hline $\mathrm{F}$ & $167(55.9)$ & $165(56.3)$ & $2(33.3)$ \\
\hline \multicolumn{4}{|l|}{ Underlying conditions } \\
\hline Diabetes & $1(0.3)$ & $1(0.3)$ & $0(0.0)$ \\
\hline Hypertension & $6(2.0)$ & $6(2.0)$ & $0(0.0)$ \\
\hline Asthma & $1(0.3)$ & $1(0.3)$ & $0(0.0)$ \\
\hline Coronary artery disease & $1(0.3)$ & $1(0.3)$ & $0(0.0)$ \\
\hline Cancer & $3(1.0)$ & $3(1.0)$ & $0(0.0)$ \\
\hline Connective tissue disease & $1(0.3)$ & $1(0.3)$ & $0(0.0)$ \\
\hline Liver disease & $1(0.3)$ & $1(0.3)$ & $0(0.0)$ \\
\hline Thyroid disease & $2(0.7)$ & $2(0.7)$ & $0(0.0)$ \\
\hline Current pregnancy & $4(1.4)$ & $4(1.4)$ & $0(0.0)$ \\
\hline \multicolumn{4}{|c|}{ Charlson Comorbidity Index score } \\
\hline 0 & $287(96.0)$ & $281(95.9)$ & $6(100.0)$ \\
\hline 1 & $8(2.7)$ & $8(2.7)$ & $0(0.0)$ \\
\hline$\geq 2$ & $4(1.3)$ & $4(1.4)$ & $0(0.0)$ \\
\hline
\end{tabular}

public transportation to get to the airport, it is highly likely that her infection was transmitted in the flight via indirect contact with an asymptomatic patient. She reported coughing, rhinorrhea, and myalgia on quarantine day 8 and was transferred to a hospital on quarantine day 14. The remaining 292 passengers were released from quarantine on day 15.

All crew members $(n=10)$ and medical staff dispatched from KCDC $(n=8)$ were quarantined at a government quarantine facility for 2 weeks and were tested twice for SARS-CoV-2, on quarantine days 1 and 14. All 18 members of the cabin crew and medical staff were negative for SARS-CoV-2 on both occasions.

To reinforce our results, we performed an external validation using a different dataset. Another evacuation flight of 205 passengers from Milan, Italy, to South Korea on April 3, 2020, was also conducted by KCDC under strict infection control procedures. Among the passengers on this flight were 3 asymptomatic patients who tested positive on quarantine day 1 and 1 patient who tested negative on quarantine day 1 and positive on quarantine day 14 . On the basis of an epidemiologic investigation, the authors and KCDC suspect that this infection was also transmitted by inflight contact.

\section{Conclusions}

This study was one of the earliest to assess asymptomatic transmission of COVID-19 on an aircraft. Previous studies of inflight transmission of other respiratory infectious diseases, such as influenza and severe acute respiratory syndrome, revealed that sitting near a person with a respiratory infectious disease is a major risk factor for transmission $(6,7)$, similar to our own findings. Considering the difficulty of airborne infection transmission inflight because of highefficiency particulate-arresting filters used in aircraft ventilation systems, contact with contaminated surfaces or infected persons when boarding, moving, or disembarking from the aircraft may play a critical role in inflight transmission of infectious diseases $(6,7)$.

Previous studies reported that viral shedding can begin before the appearance of COVID-19 symptoms $(8,9)$, and evidence of transmission from presymptomatic and asymptomatic persons has been reported in epidemiologic studies of SARS-CoV-2 $(5,10,11)$. Because KCDC performed strong infection control procedures during boarding; the medical staff and crew members were trained in infection control; all passengers, medical staff, and crew members were tested twice for SARS-CoV-2; and a precise epidemiologic investigation was conducted, the most plausible explanation for the transmission of SARS-CoV-2 to a passenger on the aircraft is that she became infected by an asymptomatic but infected passenger while using an onboard toilet. Other, less likely, explanations for the transmission are previous SARS-CoV-2 exposure, longer incubation period, and other unevaluated situations.

The control measures incorporated into our cohort study provide a higher level of evidence than previous studies on asymptomatic transmission $(5,10,11)$. Our findings suggest the following strategies for the prevention of SARS-CoV-2 transmission on an aircraft. First, masks should be worn during the flight. Second, because contact with contaminated surfaces increases the risk for transmission of 
A

Flight from

Milan, Italy

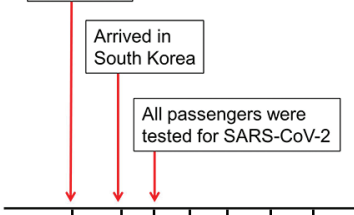

$\begin{array}{lllllllllllllllll}31 & 1 & 2 & 3 & 4 & 5 & 6 & 7 & 8 & 9 & 10 & 11 & 12 & 13 & 14 & 15 & 16\end{array}$ March

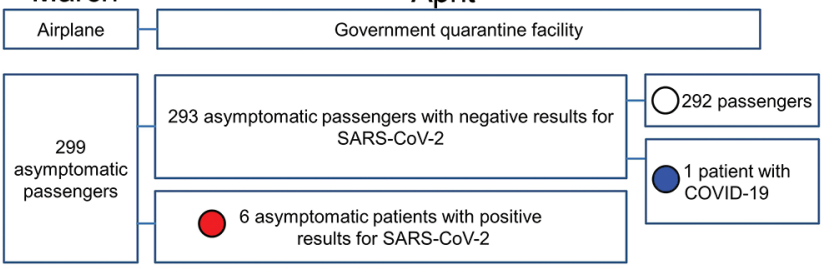

C
All passengers were tested once again for SARS-CoV-2
Patient (28/F) with a positive result for SARS-CoV-2 on April 15, 2020

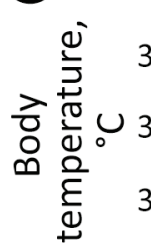

Airplane Government quarantine facility

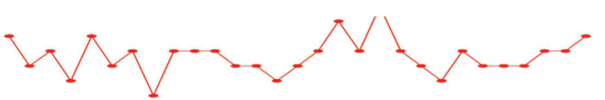

36.0

B

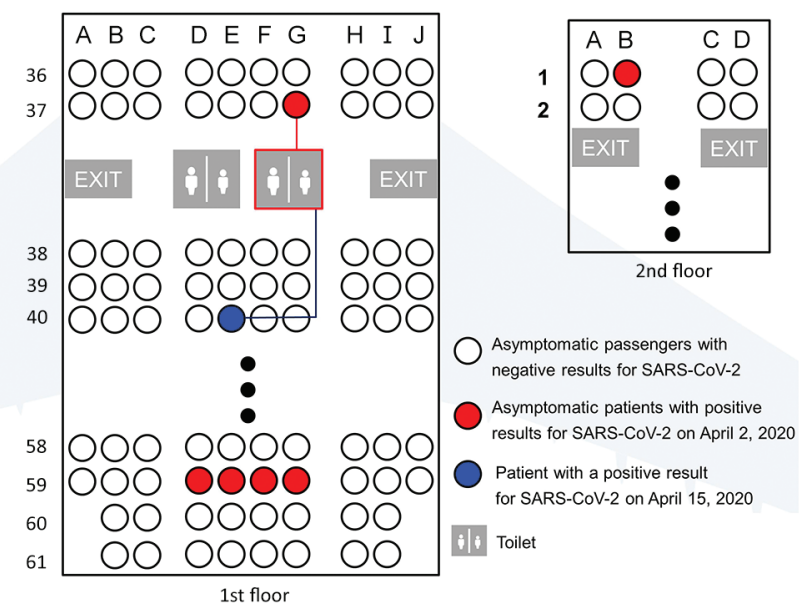

Figure. Cohort study of severe acute respiratory syndrome coronavirus 2 (SARS-CoV-2) transmission on evacuation flight from Milan, Italy, to Incheon, South Korea, on March 31, 2020. Red indicates asymptomatic patients who tested positive on quarantine day 1 (April 1, 2020); blue indicates asymptomatic patient who tested positive on quarantine day 14 (April 15, 2020). A) Timeline of flight, passenger quarantine, and testing protocol. B) Location of 6 asymptomatic patients and subsequently infected patient on flight. C) Case report of patient apparently infected during evacuation flight.

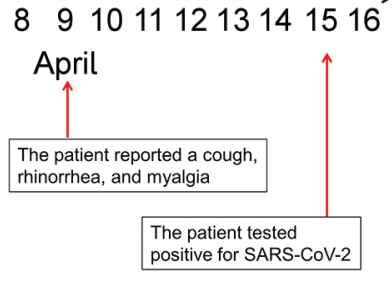

\section{About the Author}

SARS-CoV-2 among passengers, hand hygiene is necessary to prevent infections. Third, physical distance should be maintained before boarding and after disembarking from the aircraft.

Our research provides evidence of asymptomatic transmission of COVID-19 on an airplane. Further attention is warranted to reduce the transmission of COVID-19 on aircraft. Our results suggest that stringent global regulations for the prevention of COVID-19 transmission on aircraft can prevent public health emergencies.

\section{Acknowledgments}

We thank the Korea Centers for Disease Control and

Prevention for its support of this study.

This work was supported by the National Research Foundation of Korea (NRF) grant funded by the government of Korea (grant no. NRF2019R1G1A109977912). The funders had no role in study design, data collection, data analysis, data interpretation, or writing of the report.
Dr. Bae is a physician with the Soonchunhyang University College of Medicine, Seoul, South Korea. His research interests are emerging infectious diseases and radiology.

\section{References}

1. Yang N, Shen Y, Shi C, Ma AHY, Zhang X, Jian X, et al. In-flight transmission cluster of COVID-19: a retrospective case series. Infect Dis (Lond). 2020 Jul 31:1-11.

2. Ng O-T, Marimuthu K, Chia P-Y, Koh V, Chiew CJ, De Wang L, et al. SARS-CoV-2 infection among travelers returning from Wuhan, China. N Engl J Med. 2020;382: 1476-8. https://doi.org/10.1056/NEJMc2003100

3. Lee SW, Ha EK, Yeniova AÖ, Moon SY, Kim SY, Koh HY, et al. Severe clinical outcomes of COVID-19 associated with proton pump inhibitors: a nationwide cohort study with propensity score matching. Gut. 2020;gutjnl-2020-322248. https:// doi.org/10.1136/gutjnl-2020-322248

4. World Health Organization. Operational considerations for managing COVID-19 cases or outbreaks on board ships: interim guidance, 25 March 2020. World Health Organization; 2020 [cited 2020 Aug 13]. https:/ / apps.who. int/iris/bitstream/handle/10665/331591/WHO-2019-nCoVShips-2020.2-eng.pdf 
5. Arons MM, Hatfield KM, Reddy SC, Kimball A, James A, Jacobs JR, et al.; Public Health-Seattle and King County and CDC COVID-19 Investigation Team. Presymptomatic SARS-CoV-2 infections and transmission in a skilled nursing facility. N Engl J Med. 2020;382:2081-90. https:/ / doi.org/10.1056/NEJMoa2008457

6. Olsen SJ, Chang H-L, Cheung TY-Y, Tang AF-Y, Fisk TL, Ooi SP-L, et al. Transmission of the severe acute respiratory syndrome on aircraft. N Engl J Med. 2003;349:2416-22. https://doi.org/10.1056/NEJMoa031349

7. Mangili A, Gendreau MA. Transmission of infectious diseases during commercial air travel. Lancet. 2005;365:98996. https:// doi.org/10.1016/S0140-6736(05)71089-8

8. He X, Lau EHY, Wu P, Deng X, Wang J, Hao X, et al. Temporal dynamics in viral shedding and transmissibility of COVID-19. Nat Med. 2020;26:672-5. https:// doi.org/ $10.1038 /$ s41591-020-0869-5
9. Choi S-H, Kim HW, Kang J-M, Kim DH, Cho EY

Epidemiology and clinical features of coronavirus disease 2019 in children. Clin Exp Pediatr. 2020;63:125-32. https:/ / doi.org/10.3345/cep.2020.00535

10. Song J-Y, Yun J-G, Noh J-Y, Cheong H-J, Kim W-J. Covid-19 in South Korea - challenges of subclinical manifestations. N Engl J Med. 2020;382:1858-9. https://doi.org/10.1056/ NEJMc2001801

11. Bai Y, Yao L, Wei T, Tian F, Jin D-Y, Chen L, et al. Presumed asymptomatic carrier transmission of COVID-19. JAMA. 2020;323:1406. https://doi.org/10.1001/jama.2020.2565

Address for correspondence: Dong Keon Yon, Armed Force Medical Command, Republic of Korea Armed Forces, 81

Saemaeul-ro 177, Seongnam, Gyeonggi-do, 463-040, South Korea; email:yonkkang@gmail.com

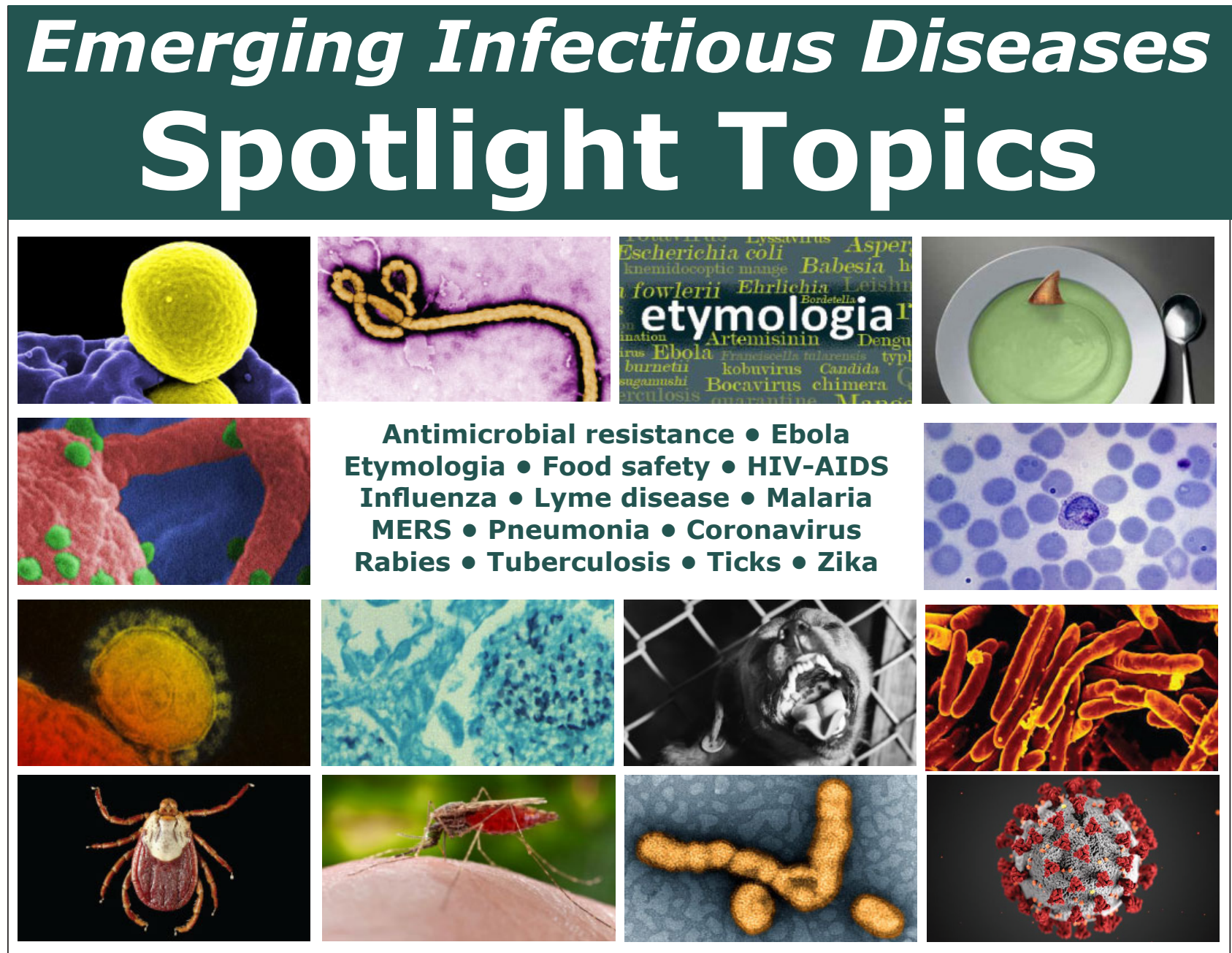

EID's spotlight topics highlight the latest articles and information on emerging infectious disease topics in our global community https:/ / wwwnc.cdc.gov/eid/page/spotlight-topics 